\title{
Siedlungswasserwirtschaftliche Strukturtypen und ihre Potenziale für die dezentrale Bewirtschaftung von Niederschlagswasser
}

\author{
Lena Simperler · Paul Himmelbauer · Gernot Stöglehner · Thomas Ertl
}

Online publiziert: 5. September 2018

(C) Der/die Autor(en) 2018

Zusammenfassung In der Bewirtschaftung von Niederschlagswasser setzt sich in den letzten Jahren ein Trend in Richtung dezentraler, flexibler Lösungen und grüner Infrastruktur durch, um besser an künftige Herausforderungen wie den Klimawandel oder die Urbanisierung angepasst zu sein. Bei der Erweiterung oder Neuerschließungen von Siedlungsgebieten wird zunehmend der Erhalt des natürlichen Wasserhaushalts angestrebt. Hier fällt es vergleichsweise leicht, dezentrale Maßnahmen wie Versickerung oder Retention entsprechend dem Stand der Technik und Forschung vorzuschreiben bzw. umzusetzen.

Innerhalb bestehender Stadt- und Siedlungsstrukturen ist eine vertiefte Auseinandersetzung mit Bebauung, Flächennutzung und Flächenwidmung erforderlich, um mögliche geeignete Standorte für die Niederschlagswasserbewirtschaftung $\mathrm{zu}$ identifizieren. Es wird eine Methode aufgezeigt, um auf Basis bestehender amtlicher Daten räumliche Strukturtypen aus Sicht der Siedlungswasserwirtschaft abzugrenzen. Diese dienen als Basis, um flächenbezogen innerhalb des Bestands Potenziale und Restriktionen für die Niederschlagswasserbewirtschaftung zu identifizieren. Die hier entwickelte Methodik kann einen Beitrag zur fun-

\section{L. Simperler .}

Univ.-Prof. DI Dr. T. Ertl

Institut für Siedlungswasserbau, Industriewasserwirtschaft und Gewässerschutz, Universität für Bodenkultur Wien,

Muthgasse 18, 1190 Wien, Österreich

DI P. Himmelbauer $(\bowtie)$.

Univ.-Prof. DI Dr. G. Stöglehner

Institut für Raumplanung,

Umweltplanung und Bodenordnung,

Universität für Bodenkultur Wien,

Peter-Jordan-Straße 82, 1190 Wien,

Österreich

paul.himmelbauer@boku.ac.at dierten Entscheidungsfindung für die Auswahl und Umsetzung von dezentralen Maßnahmen der Niederschlagswasserbewirtschaftung leisten.

\section{Urban structures and their potential for decentralized stormwater management}

Abstract The implementation of decentralized flexible measures and green infrastructure for stormwater management is increasing, in order to better adapt to future challenges for example to climate change or urbanization. For settlement expansions and new constructions maintaining the natural water balance is gaining importance for stormwater management. In these areas realizing decentralized state-of-theart measures is relatively simple and can also be enforced through regulative measures.

In existing urban structures, however, a detailed analysis of the built structure, the land use and zoning is necessary in order to identify potential locations for decentralized measures for stormwater management. In this paper a method is presented on how urban structures can be categorized from a stormwater management perspective based on nationwide available official data. The defined urban structures are used to identify potentials and constraints for stormwater management. The developed method can contribute to sound decision making regarding the selection and implementation of decentralized measures for stormwater management.

\section{Hintergrund und Zielsetzung}

In der Raum- und Infrastrukturplanung stellen sich stetig ändernde Rahmenbedingungen, wie der Klimaschutz und die Anpassung an den Klimawandel, die zunehmende Urbanisierung und der demografische Wandel als zentra- le Herausforderungen dar. Zukünftige Themenstellungen umfassen die Bearbeitung von räumlichen und baulichen Herausforderungen in einem regionalen Kontext, der über die Verwaltungsgrenzen von Gemeinden hinausgeht mit einem größeren Fokus auf Funktionsräume (z.B. Gebiete für die Versorgung mit erneuerbaren Energiequellen, einzugsgebietsbezogene Betrachtung von Hoch- und Hangwasser, interkommunale Betriebsgebiete) ebenso wie die Finanzierung, Instandhaltung bzw. Adaption von Infrastrukturen. Angestrebt wird die Entwicklung von robusten und anpassungsfähigen Raumstrukturen sowie deren Versorgung mit dezentralen und flexiblen Infrastruktursystemen (ARL 2017).

Da Entscheidungen und bauliche Maßnahmen in der Siedlungswasserwirtschaft über viele Jahrzehnte bis Jahrhunderte wirken, gilt es auch in deren Planung mittel- bis langfristige Aspekte wie zukünftige Erweiterungsgebiete von Gemeinden sowie die Verfügbarkeit von Flächen für dezentrale Infrastrukturen mit in die Entscheidungsfindung einzubeziehen. Es gilt, jene Standorte bzw. Maßnahmen auszuwählen, die auch unter veränderten und unterschiedlichen Bedingungen langfristig ihre Wirkung entfalten können (Heiland et al. 2017).

$\mathrm{Zu}$ diesen zählen beispielsweise dezentrale Maßnahmen für die Niederschlagswasserbewirtschaftung (NWB), für welche das Interesse aufgrund ihrer Flexibilität im Einsatz und vielseitiger Vorteile seit etwa zehn Jahren steigt: Neben dem Überflutungsschutz kann vor allem grüne Infrastruktur die Resilienz von räumlichen Strukturen erhöhen, die natürliche Wasserbilanz wiederherstellen, $\mathrm{zu}$ einem verbesserten Mikroklima beitragen sowie gesundheitliche oder soziale Mehrwerte erzielen (Braubach et al. 2017; Fletcher et al. 2015). Im Zusammenhang mit der Klimawandelanpassung spielt der 
Beitrag von grüner Infrastruktur auch eine besondere Rolle im Bereich einer integrierten räumlichen Planung (ARL 2017), beispielsweise zur Minderung von urbanen Hitzeinseln (Matzinger et al. 2017).

Während in der Vergangenheit Niederschlagswasser (NW) in Österreich in vielen Gemeinden vor allem über die Kanalisation abgeleitet wurde, ist in den letzten Jahren im Neubau vermehrt auf den Einsatz von Versickerungsanlagen gesetzt worden (Assmann et al. 2015). Da die direkte Ableitung von NW ins Gewässer negative Folgen für den Wasserhaushalt (Burns et al. 2012), das Stadtklima und die Gewässerqualität haben kann (Matzinger et al. 2017), werden vor allem für Neubaugebiete alternative Maßnahmen diskutiert. Während dies in Österreich noch keinen Einfluss auf die Gesetzgebung hat, ist beispielsweise in Deutschland ein Anhang zum Wasserhaushaltsgesetz in Entwurf, welcher den Erhalt des natürlichen Wasserhaushalts bei entwässerungstechnischen Neuerschließungen vorschreibt (DWA und BWK 2016).

Bei der Entwicklung und Erschließung von neuen Siedlungsgebieten können Aspekte der NWB jedoch bereits frühzeitig im Rahmen der Flächenwidmungs- und Bebauungsplanung integriert werden. Dadurch können bereits vor jeder Bebauung die für dezentrale grüne Infrastruktur notwendigen Flächen gesichert werden. Je nach Bundesland bestehen in Österreich unterschiedliche Möglichkeiten im Rahmen der örtlichen Raumplanung, Flächen im Flächenwidmungsplan zu sichern und den Umgang mit NW im Rahmen von Bebauungsplänen zu regeln. Beispielsweise können in Niederösterreich im Bebauungsplan Zonen definiert werden, in denen die Ableitung von Niederschlagswasser in Kanäle und/oder Vorfluter untersagt bzw. als eingeschränkt zulässig befunden wird (§ 30 (2) NÖ ROG 2014).

Bei Sanierungsmaßnahmen oder überlasteten Kanalnetzen kann aber auch im Bestand eine Adaptierung des Entwässerungssystems notwendig sein. Auch hier kommen dezentrale Systeme zum Einsatz. Neben natürlichen Randbedingungen, wie beispielsweise die Sickerfähigkeit des Untergrundes, der Grundwasserstand oder die Topografie (Grimm und Achleitner 2010), hat auch die bestehende Bebauung Einfluss auf die Umsetzbarkeit unterschiedlicher Maßnahmen.
Besonders bei dezentralen NWBMaßnahmen, welche an der Oberfläche umgesetzt werden, stellen Instrumente der örtlichen Raumplanung und die bestehenden baulich-räumlichen Strukturen wichtige Rahmenbedingungen für die Anwendbarkeit dieser Maßnahmen dar. Örtliche Entwicklungskonzepte enthalten in der Regel jene Flächen, die in einer Gemeinde für die mittel- bis langfristige Entwicklung (z.B. Wohngebiete, Betriebsgebiete) vorgesehen sind. Während über die Flächenwidmung geregelt ist, welche Nutzungen im Bereich von Bauland und Grünland zulässig sind, und wie deren Erschließung erfolgt (Verkehrsflächen), können Bebauungspläne konkrete Rahmenbedingungen der Bebauung festlegen, die einen Einfluss auf Ableitung bzw. Versickerung von NW nehmen. Weiters kann die örtliche Raumplanung eine Datenbasis für bebautes Bauland, Lücken innerhalb des Baulandes oder leerstehende Gebäude bieten, Informationen, die für die Identifikation von möglichen Standorten dezentraler NWB relevant sind.

Eine große Herausforderung ist es, innerhalb bestehender Stadt- bzw. Siedlungsstrukturen, Flächenwidmungsund Bebauungsplänen Flächen für die NWB bereitzustellen. Umso mehr ist eine umfassende räumliche Auseinandersetzung mit Nutzungen, Bebauung, Erschließung und Versorgung mit Infrastrukturen sowie mit Standorten von Sondernutzungen und öffentlichen Einrichtungen erforderlich, um mögliche Standorte für die NWB innerhalb bestehender Stadt- bzw. Siedlungsgebiete $\mathrm{zu}$ identifizieren. Eine solche Analyse auf Basis unterschiedlicher räumlicher Strukturtypen kommt in der Stadtplanung (Kuzmich et al. 2011), Energieraumplanung (Hegger et al. 2012; Stoeglehner et al. 2016), Stadtklimatologie und Klimawandelanpassung zur Anwendung (Buchholz et al. 2016; Gasienica-Wawrytko et al. 2014; Kruse et al. 2014; Stewart und Oke 2012).

Die Einteilung in Strukturtypen dient zur Abschätzung von Potenzialen, Restriktionen und Bedürfnissen eines räumlich zusammenhängenden Gebiets mit ähnlichen Merkmalen. Abwasserverbände oder Gemeinden können diese Informationen nutzen, um jene Standorte auszuwählen, an welchen prioritär Maßnahmen der NWB gesetzt werden sollen. In der Klimawandelanpassung wird beispielsweise die Anpassungsfähigkeit einzelner
Strukturtypen in Bezug auf Temperaturerhöhung und Veränderungen beim Niederschlag ermittelt, um gesamtheitliche Konzepte für Stadtteile erarbeiten zu können (Kruse et al. 2014).

Das Ziel dieses Artikels ist es, eine Methodik zur Abgrenzung siedlungswasserwirtschaftlich relevanter Strukturtypen zu erarbeiten und deren Potenziale und Restriktionen in Bezug auf den Einsatz dezentraler NWB-Maßnahmen $\mathrm{zu}$ ermitteln. Es wird aufgezeigt und diskutiert, wie diese Typisierung über flächendeckend für Österreich verfügbare amtliche Daten hergestellt werden kann.

Diese soll Abwasserverbände und Gemeinden in Österreich dabei unterstützen, die Potenziale und Restriktionen für den Einsatz von dezentralen NWB-Maßnahmen in bestehenden Stadt- und Siedlungsgebieten abzuschätzen. Die Strukturtypen sollen eine strategische Entscheidungsgrundlage für den Umgang mit NW auf örtlicher Ebene liefern. Sowohl für die Adaption bzw. Erneuerung der Infrastruktur in bestehende Siedlungen als auch für die Planung von Erweiterungsgebieten bieten sie einen Rahmen, um zu einer integrierten Raum- und Infrastrukturplanung beizutragen.

\section{Methodik}

Die Ermittlung von Merkmalen, welche für die NWB relevant sind, stellt den Grundstein für die Abgrenzung der einzelnen Strukturtypen dar. Die methodische Vorgangsweise zur Identifikation von Strukturtypen und Kriterien sowie deren Anwendbarkeit auf Basis in Österreich verfügbarer Daten ist in Abb. 1 dargestellt. Hierfür wurde zu Beginn eine systematische Literaturrecherche bestehender Abgrenzungen von Strukturtypen durchgeführt. Diese umfasst die Suche in der Literaturdatenbank Scopus sowie die Recherche in nationalen und internationalen Forschungsberichten.

In einem zweiten Schritt wurden die Abgrenzungskriterien der Strukturtypen kategorisiert und in Bezug auf die Relevanz für die NWB priorisiert. Diese Kriterien umfassten z.B. Bebauungsgrad, Geschossflächenzahl, Anzahl der Geschosse, Anzahl/Dichte der EinwohnerInnen, Anzahl der Stellplätze, Anteile bebauter/versiegelter/teilversiegelter Flächen, Art der Flächennutzung (Wohnen, Gewerbe, Büro etc.), Eigentums- 


\begin{tabular}{|c|c|c|c|}
\hline $\begin{array}{l}\text { Literaturrecherche } \\
\text { zu Strukturtypen }\end{array}$ & $\begin{array}{l}\text { Kategorisi } \\
\text { Priorisieru } \\
\text { Kriterien }\end{array}$ & $\begin{array}{l}\text { ng und } \\
\text { von }\end{array}$ & $\begin{array}{l}\text { Datenverfügbarkeit in } \\
\text { Österreich }\end{array}$ \\
\hline \multirow{4}{*}{$\begin{array}{l}\text { Siedlungswasser- } \\
\text { wirtschaft } \\
\text { Klimawandel- } \\
\text { anpassung } \\
\text { Energieraum- } \\
\text { planung } \\
\text { Stadtklimatologie }\end{array}$} & & Kriterien & \multirow{4}{*}{$\begin{array}{l}\text { - Open Data - data.gv.at. } \\
\text { - Statistik Austria } \\
\text { - Bundesamt für Eich- und } \\
\text { Vermessungswesen } \\
\text { - Datenportale und Web- } \\
\text { GIS der Bundesländer } \\
\text { - ÖROK Atlas }\end{array}$} \\
\hline & $\begin{array}{l}\text { Struktur- } \\
\text { typ } 1\end{array}$ & & \\
\hline & $\begin{array}{l}\text { Struktur- } \\
\text { typ } 2\end{array}$ & & \\
\hline & $\begin{array}{l}\text { Struktur- } \\
\text { typ .... }\end{array}$ & & \\
\hline
\end{tabular}

Abb. 1 Schematische Vorgangsweise bei der Kriterienwahl

form, Dachform, Dachneigung und Material sowie Flächenwidmung.

Die relevanten Abgrenzungskriterien wurden im Anschluss auf die flächendeckende Datenverfügbarkeit in Österreich geprüft. Folgende Grundlagen können für die Abgrenzung herangezogen werden:

- das Gebäude- und Wohnungsregister (GWR) als Basis für Informationen zur Bebauungsstruktur, Nutzungen und EigentümerInnen von Gebäuden sowie Daten zu EinwohnerInnen,

- die Flächenwidmung als Basis für die Unterscheidung verschiedener Nutzungen im Siedlungsbereich,

- die digitale Katastralmappe (DKM) als ergänzende Grundlage für Bebauung, Flächennutzungen und Verkehrserschließung und

- das Integrierte Verwaltungs- und Kontrollsystem (INVEKOS) als Datenbasis für landwirtschaftliche Hofstellen sowie landwirtschaftliche Nutzungen.

Generell erfolgt eine Aggregation der unterschiedlichen Daten auf Ebene von Baublöcken mit derselben Flächenwidmung. Die relevanten Merkmale werden im Detail im folgenden Kapitel beschrieben.

Basierend auf Kriterien aus der herangezogenen Literatur wurden die einzelnen Merkmale in mehrere Klassen unterteilt. Diese Unterteilungen bilden die Grundlage für die Definition der Strukturtypen mit unterschiedlichen Merkmalsausprägungen. Die Vorgangsweise ist schematisch in Abb. 2 dargestellt.

Im Anschluss wurden die Potenziale und Restriktionen, welche sich aus den Daten ableiten lassen, zusammengefasst und um Erkenntnisse aus der Klimawandelanpassung ergänzt.

\section{Siedlungswasserwirtschaftlich relevante Kriterien und Indikatoren}

Im Zuge der Literaturrecherche wurden vier Merkmale bestimmt, welche für die NWB relevant sind. Die Merkmale stellen Kriterien und Indikatoren für die Abgrenzung der Strukturtypen dar. Im Folgenden werden die Merkmale erläutert und ihre Relevanz für die Anwendbarkeit von NWB-Maßnahmen beschrieben.

\subsection{Bodenversiegelung durch Bebauung}

Prinzipiell kann bei der Versiegelung innerhalb eines Blocks zwischen bebauten Flächen durch Gebäude und befestigten Flächen, wie Terrassen, Stellplätzen und Wegen unterschieden werden (Grimm und Achleitner 2010). Da Daten über befestigte Flächen auf Grundstücksebene nicht flächendeckend verfügbar sind, wird bei der Klas- sifizierung des Versiegelungsgrades von Baublöcken nur die mit Gebäuden bebaute Fläche berücksichtigt. Datenbasis hierfür bildet die Bruttogrundfläche des Gebäudes, welche im GWR erfasst ist sowie die Grundstücksfläche, welche aus der DKM stammt. Die gewählten Kategorien des Bebauungsgrades sind in Tab. 1 zusammengefasst. Die qualitative Beschreibung findet sich zum Teil in der Beschreibung der Strukturtypen wieder.

Da Maßnahmen der NWB, welche an der Oberfläche ausgeführt werden, einen Flächenbedarf haben, welcher zwischen wenigen Prozent der angeschlossen Fläche bis zu über $50 \%$ variieren kann (Matzinger et al. 2017), stellt die Versiegelung eines Baublocks ein wichtiges Kriterium für der Beurteilung der Einsatzbarkeit einzelner NWBMaßnahmen dar.

\subsection{Geschossflächenzahl}

Die Geschossflächenzahl ist ein Merkmal für die bauliche Ausnutzung eines Grundstücks. Sie wird ermittelt als Verhältnis der Geschossfläche und der Grundstücksfläche. Ergänzt um Informationen zur Flächennutzung und Siedlungsdichte (Einwohner/ha) auf Basis des GWR können mehr oder weniger dicht bebaute Gebiete voneinander unterschieden werden.

Die Geschossflächenzahl wurde in drei Kategorien unterteilt. Diese spiegeln die bauliche Dichte auf Grundstücks- bzw. Blockebene bei der Unterteilung der Strukturtypen wieder und sind in Tab. 2 dargestellt.

Mit der Zunahme der Geschossflächenzahl ist in der Regel auch eine Zunahme des Stellplatzbedarfs und der befestigten bzw. versiegelten Fläche zur inneren Erschließung (z. B. Wohnwege, Zufahrten) verbunden (Hegger et al. 2012).

Besonders Stellplätze müssen bei einer siedlungswasserwirtschaftlichen Betrachtung räumlicher Strukturen be-

\section{Aufbereitung der} Datengrundlagen

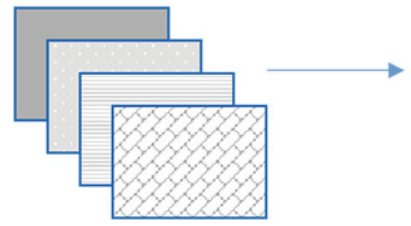

Aggregation, Analyse und Klassifikation

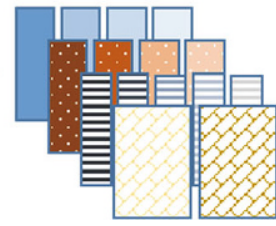

Bestimmung von Strukturtypen
1

2

3

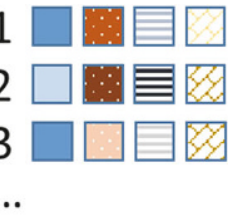

$\ldots$

Abb. 2 Schematische Vorgangsweise bei der Erstellung der Strukturtypen

Tab. 1 Kategorien des Bebauungsgrads

\begin{tabular}{|c|c|c|}
\hline \multicolumn{3}{|c|}{ Bebauungsgrad in Prozent } \\
\hline$<30 \%$ & $30-50 \%$ & $>50 \%$ \\
\hline Gering & Mittel & Hoch \\
\hline
\end{tabular}


Tab. 2 Kategorien der Dichte

\begin{tabular}{lll}
\multicolumn{2}{l}{ Geschossflächenzahl } & \\
$<0,3$ & $0,3-0,6$ & $>0,6$ \\
Gering & Mittel & Hoch
\end{tabular}

Definition: Verhältnis der Brutto-geschossflächen zur Grundstücksfläche

rücksichtigt werden, da diese einen Einfluss auf die Abflussqualität des NW haben (ÖWAV-Regelblatt 45 2014). Das ÖWAV-Regelblatt 35 (2003) und ÖWAV-Regelblatt 45 (2014) unterscheiden dabei zwischen unterschiedlichen Kategorien an Stellplätzen in Bezug auf Stellplatzanzahl und Häufigkeit des Stellplatzwechsels. Je nach Kategorie liegen Einschränkungen der Anwendbarkeit einzelner Maßnahmen vor. Je nach Bundesland ist die Mindestanzahl an Stellplätzen (z.B. je Wohnung, pro $\mathrm{m}^{2}$ Verkaufsfläche etc.) im Rahmen von Gesetzen oder Verordnungen definiert.

Im Zusammenhang mit der Nutzung von NW spielt das Wissen über die Anzahl potenzieller VerbraucherInnen eine wichtige Rolle, da der Bedarf an Brauchwasser unter anderem in die Dimensionierung von Speicherbehältern einfließt (Woods Ballard et al. 2015). Die Geschossflächenzahl liefert als Indikator für die Dichte somit eine Grundlage für die Bewertung der NW-Nutzung.

\subsection{Flächennutzung und Flächenwidmung}

Die Flächennutzung wird aus einer Verschneidung von Informationen aus DKM, GWR und INVEKOS-Daten abgeleitet (Tab. 3) und um die Flächenwidmung als Indikator für mögliche Nutzungen ergänzt. Dabei kann zwischen Wohnen, Mischnutzung, Büros,
Gewerbe, Industrie und Landwirtschaft unterschieden werden.

Die Qualität des Oberflächenabflusses lässt sich flächendeckend am besten über die Nutzung der jeweiligen Fläche ableiten. Je nach Nutzung kann eine unterschiedliche Beeinträchtigung des Oberflächenabflusses vorliegen. Da der Gewässer-, Boden- und Grundwasserschutz ein Ziel der Siedlungsentwässerung darstellt (EN 752 2017), muss die Oberflächenverschmutzung eines Baublocks in die Planung einfließen. Je nach Verschmutzungsgrad wird im ÖWAV-Regelblatt 35 (2003) und im ÖWAV-Regelblatt 45 (2014) der Einsatz unterschiedlicher Maßnahmen und Reinigungsstufen empfohlen. $\mathrm{Zu}$ den wichtigsten Merkmalen auf Baublockebene, welche sich über die Nutzung ableiten lassen, gehören die Anzahl an Stellplätzen und die Häufigkeit des Fahrzeugwechsels sowie der Verschmutzungsgrad von Hofflächen.

Die Flächenwidmung spielt beim Einsatz dezentraler Maßnahmen zur Entwässerung eine besondere Rolle, da sie über die unterschiedlichen Widmungskategorien bestimmt, welche Nutzungen im jeweiligen Gebiet zulässig sind. Zur Abschätzung von Potenzialen und Restriktionen sowie zur Auswahl von Maßnahmen und Standorten für die NWB sind nicht nur die aktuellen Nutzungen relevant, sondern auch die Information, welche Nutzungen auf Basis der Flächenwidmung zukünftig dort möglich sind. Diese Möglichkeit der Nutzungsänderung sollte bei der Maßnahmenplanung mitbedacht werden. So können beispielsweise als Kerngebiet gewidmete Flächen, welche zum Zeitpunkt der Adaption des Entwässerungssystems für Wohnzwecke genutzt werden, zu einem späteren Zeitpunkt auch betrieblich genutzt werden, und damit beispielsweise einen höheren Stellplatzwechsel bzw. eine stärkere Verschmutzung des NW zur Folge haben.

Weiters kann auch für die Regenwassernutzung die Art der NutzerInnen eine Rolle spielen. Hierbei kann die Unterscheidung zwischen reinen Wohngebieten, Kerngebieten mit Mischnutzung oder etwa reinen Bürogebieten von Relevanz sein, diese Information kann aus den hier angeführten Datenquellen generiert werden.

\subsection{EigentümerInnen}

Hinsichtlich EigentümerInnentypen lässt das GWR auf Gebäudeebene folgende Unterscheidung zu: Privatperson(en), Bund, Land, Gemeinde, andere öffentlich (rechtliche) Körperschaft, gemeinnützige Bauvereinigung, sonstiges Unternehmen, anderer Eigentümer, nicht bekannt. Diese lassen sich in drei Gruppen teilen, welche für die NWB relevant sind:

- Die öffentliche Hand (Bund, Länder, Gemeinden, andere öffentlich (rechtliche) Körperschaften). Diese hat hohe Wirkmächtigkeit auf den eigenen Flächen.

- EigentümerInnen mit hohem individuellem Handlungsspielraum, welche auch eine hohe Wirkmächtigkeit entfalten können. Zu dieser Gruppe zählen beispielsweise gemeinnützige Bauvereinigungen und EigentümerInnen großer zusammenhängender Flächen.

- EigentümerInnen mit geringer Wirkmächtigkeit oder geringem individuellem Handlungsspielraum. $\mathrm{Zu}$

\section{Tab. 3 Daten zu Flächennutzung und Flächenwidmung}

\begin{tabular}{l|l} 
Digitale Katastermappe & $\begin{array}{l}\text { In der DKM wird eine Unterscheidung zwischen 27 Nutzungen vorgenommen. Diese Daten liegen flächendeckend für Ös- } \\
\text { terreich mit unterschiedlicher Aktualität vor. Für die NWB können folgende Nutzungen im Siedlungsbereich relevant sein: } \\
\text { Gebäude, Gebäudenebenflächen, Betriebsflächen, Gärten, Freizeitflächen, Friedhöfe, Straßenverkehrsanlagen, Verkehrs- } \\
\text { randflächen, Parkplätze, Forststraßen, Schienenverkehrsanlagen }\end{array}$ \\
\hline Gebäude- und Wohnungsregister & $\begin{array}{l}\text { Im GWR werden 15 Gebäudeeigenschaften unterschieden. Diese können in folgende relevante Gruppen zusammenge- } \\
\text { fasst werden: Gebäude mit einer Wohnung, Gebäude mit mehreren Wohnungen, Gebäude ohne Wohnnutzung und ohne } \\
\text { Produktion, Industrie- und Lagergebäude, landwirtschaftliches Nutzgebäude, sonstige Gebäude }\end{array}$ \\
\hline $\begin{array}{l}\text { Integriertes Verwaltungs- und } \\
\text { Kontrollsystem }\end{array}$ & $\begin{array}{l}\text { Die von der AMA jährlich veröffentlichten INVEKOS Daten beinhalten detaillierte Daten zu unterschiedlichen landwirtschaft- } \\
\text { lichen Nutzungen sowie Punktinformationen über landwirtschaftliche Hofstellen }\end{array}$ \\
\hline Flächenwidmung & $\begin{array}{l}\text { Im Bereich der Widmung wird allgemein zwischen Bauland, Grünland und Verkehrsflächen unterschieden. Bauland kann je } \\
\text { nach Bundesland weiter differenziert werden in Wohngebiete, Kerngebiete, Mischgebiete, Betriebsgebiete, Industriegebie- } \\
\text { te, Agrargebiete u. v. m. }\end{array}$ \\
& $\begin{array}{l}\text { Generalisiert kann unterschieden werden zwischen Widmungsarten, in denen überwiegend Wohnnutzungen, in denen } \\
\text { überwiegend gemischte bauliche Nutzungsormen, in denen überwiegend betriebliche Nutzungsformen sowie sonstige } \\
\text { Widmungsarten in denen überwiegend bauliche Nutzungsformen möglich sind (ÖROK 2013) }\end{array}$ \\
\hline Bebautes Bauland & $\begin{array}{l}\text { Für die Betrachtung ist auch die Information, welche von den als Bauland gewidmeten Grundstücken tatsächlich bebaut } \\
\text { sind, relevant. Diese ist in der Regel in den Gemeinden vorhanden und wird in Form von Baulandbilanzen jährlich erhoben }\end{array}$
\end{tabular}



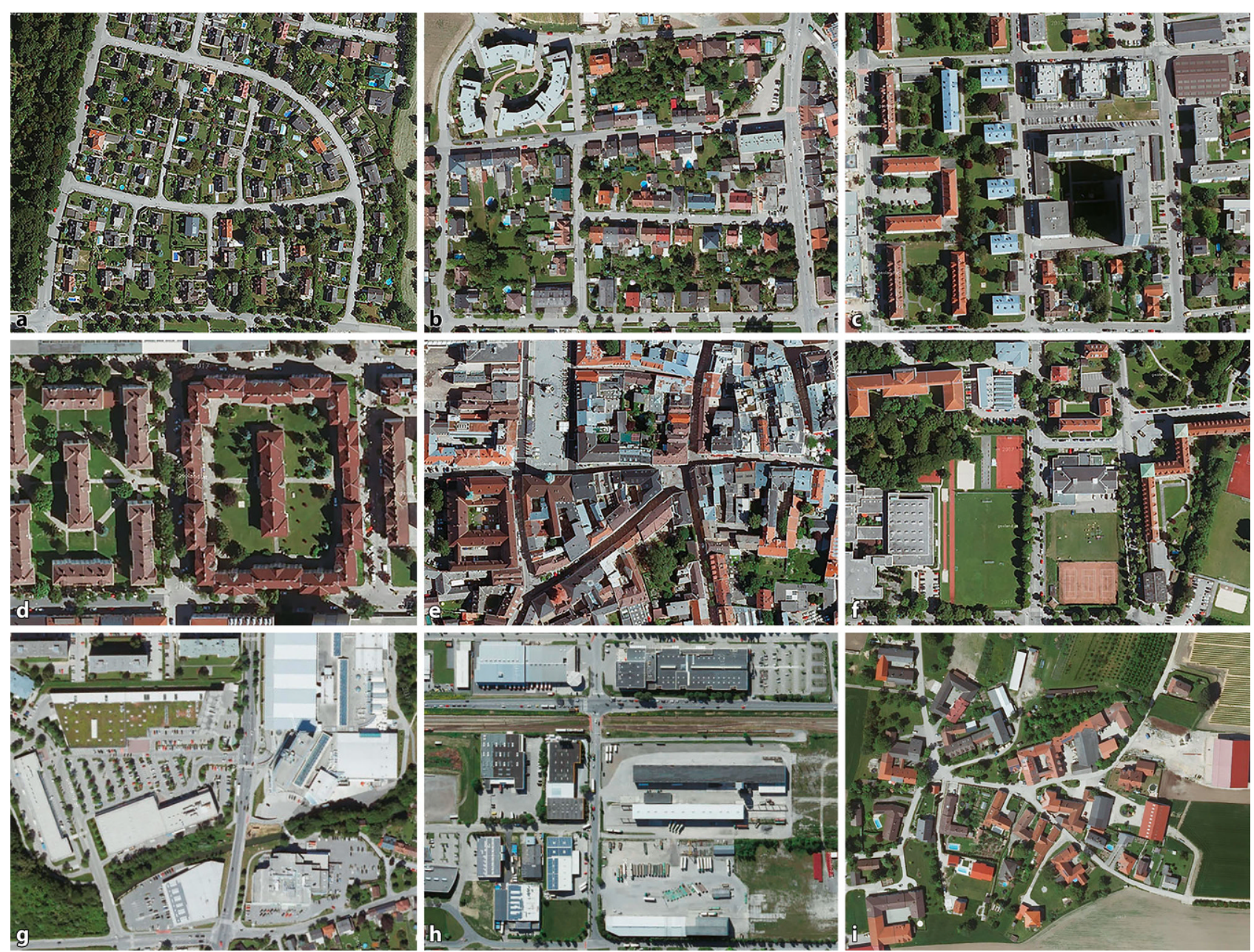

Abb. 3 Darstellung der Strukturtypen. a Gebiete mit geringer Versiegelung und Dichte, b Gebiete mit mittlerer Versiegelung und Dichte, $\mathbf{c}$ Gebiete mit mehrgeschossiger Bebauung mit mittlerer Versiegelung und hoher Dichte, d Mehrgeschossige Wohngebiete mit hoher Versiegelung und Dichte, e Stadtzentrum - Innenstadtbebauung mit hoher Versiegelung und Dichte und Mischnutzung, föffentliche Einrichtungen und Sondergebiete, $\mathbf{g}$ Büro, Verwaltung, Handel und Gewerbe, $\mathbf{h}$ Betriebsgebiete und Industrie, i dörfliche Gebiete mit landwirtschaftlichen Betrieben (Datenquelle: basemap.at)

diesen zählen unter anderem Privatpersonen in Mehrparteienhäusern oder Einfamilienhäusern.

Eigentumsverhältnisse haben nicht wie die anderen Merkmale Einfluss auf die technische Machbarkeit, sondern auf das Umsetzungspotenzial dezentraler Maßnahmen. Gerade bei Maßnahmen im Bestand, welche auf Grundstücksebene durchgeführt werden, ist das Engagement der EigentümerInnen eine Voraussetzung, da die Möglichkeiten formeller Planungsinstrumente nur beschränkt vorhanden sind. Je nach Eigentumsverhältnissen kann somit die Anzahl an Ansprechpersonen pro Gebäude oder Baublock stark variieren (Kruse et al. 2014).

Besonders bei EigentümerInnen mit hoher Wirkmächtigkeit spielt Bewusst- seinsbildung eine wichtige Rolle, da hier durch die Umsetzung von NWBMaßnahmen durch Einzelpersonen ein großer Effekt für die NWB erzielt werden kann. Für die zweite bzw. dritte EigentümerInnengruppe können $\mathrm{zu}$ sätzlich zur Bewusstseinsbildung auch Anreize oder Förderungen erforderlich sein, um diese zur Umsetzung von NBW auf Eigengrund zu gewinnen.

\section{Siedlungsstrukturtypen der NWB}

Basierend auf den zuvor beschriebenen Merkmalen können neun räumliche Strukturtypen für den Umgang mit NW in Österreich abgeleitet werden, welche sich in Bebauungsgrad, Geschossflächenzahl, Siedlungsdichte, EigentümerInnenstruktur, Flächennutzung und/ oder Widmung unterscheiden. Die Ty- pen können über die Aggregation der oben angeführten Merkmale als Basis für die Identifikation von Potenzialen und Restriktionen für die NWB abgegrenzt werden. In der Beschreibung der Typen wurden charakteristische Bauformen auf Basis bestehender Typologien (Hegger et al. 2012; Kruse et al. 2014; Müller et al. 2018) und beispielhafte Ausschnitte von Orthofotos (Abb. 3) ergänzt. Die Bezeichnungen der Typen verwenden die in Kap. 3. angeführten Untergliederungen (gering - mittel - hoch) von Bebauungsgrad (Versiegelung) und Geschossflächenzahl (Dichte).

Bei der Beurteilung der Potenziale und Restriktionen wird gleichermaßen auf den Einsatz von Maßnahmen zur offenen Ableitung, Retention und Versickerung Bezug genommen. Bei 
der offenen Ableitung sind vor allem der Platzbedarf und die Qualität des Oberflächenabflusses zu berücksichtigen. Die Möglichkeiten des Einsatzes sind auch abhängig von dem Ort, $\mathrm{zu}$ welchem das NW abgeleitet wird (ÖWAV-Regelblatt 35 2003). Bei der Retention kann zwischen darauffolgender Evapotranspiraton z.B. über ein Gründach oder offenen Wasserflächen und der NW-Nutzung unterschieden werden. Bei Gründächern ist vor allem die Dachform ausschlaggebend, während bei offenen Wasserflächen der Flächenbedarf eine wichtige Rolle spielt. Die NW-Nutzung im Haushalt wiederum ist abhängig von der Anzahl der EinwohnerInnen (Woods Ballard et al. 2015). Bei der Versickerung ist primär der Verschmutzungsgrad der Oberfläche, welche in fünf Kategorien unterteilt werden kann ( $\mathrm{F} 1$ bis F5), relevant (ÖWAV-Regelblatt 45 2014). Aber auch die Flächenverfügbarkeit spielt eine bedeutende Rolle (Grimm und Achleitner 2010). Da sich die Versiegelung, bei der Abgrenzung der Strukturtypen, nur auf die Versiegelung von Gebäuden richtet, sind gerade bei mittlerer und hoher Versiegelung zusätzliche befestigte Flächen zu bedenken.

Die neun Strukturtypen sind im Detail:

Gebiete mit geringer Versiegelung und Dichte: In diesen besteht die Bebauung überwiegend aus freistehenden Einfamilienhäusern mit 1 bis $2 \mathrm{Ge}-$ schossen. Auf Basis der Flächenwidmung bzw. GWR-Daten kann eine weitere Unterscheidung in überwiegende Wohngebiete sowie Gebiete mit zum Teil gemischter Nutzung und Bebauung (Kerngebiet, Mischgebiet) getroffen werden. In diesen ist der Anteil der befestigten Flächen, vor allem durch Stellplätze, höher als in reinen Wohngebieten. Eine Sonderform dieses Typs stellen Kleingartenanlagen dar, bei diesen variiert die Widmung je nach Bundesland. Kleingartenanlagen verfügen oft über einen zentralen Parkplatz und ein engmaschiges Wegenetz.

Aufgrund der zuvor beschriebenen siedlungswasserwirtschaftlich relevanten Kriterien lassen sich für diesen Strukturtyp die große Flächenverfügbarkeit und die überwiegend geringe Verschmutzung der Flächen als Potenziale für den Einsatz von NWB-Maßnahmen abgrenzen. Weiters kann das NW zur Bewässerung der vorhandenen Grünflächen genutzt werden. Eine Restriktion stellt die EigentümerInnen- struktur dar, da hier meist eine geringe Wirkmächtigkeit der einzelnen besteht und somit ein hoher Abstimmungsbedarf zwischen vielen EigentümerInnen vorliegt. Weiters kann bei Mischnutzung vermehrte Verschmutzung des Oberflächenabflusses durch den Fahrzeugwechsel auf Stellplätzen entstehen.

Zusätzlich zur Versiegelung durch Bebauung gibt es in diesem Strukturtyp Versiegelung von Nebenflächen durch beispielsweise Terrassen, Zufahrten oder Pools. Der unversiegelte Anteil der Flächen liegt jedoch meist bei mehr als 50\% (Buchholz und Kossmann 2015; Kuzmich et al. 2011). Da viele der dezentralen NWB-Maßnahmen einen Flächenbedarf von weniger als $10 \%$ der angeschlossenen versiegelten Fläche haben (Grimm und Achleitner 2010; Matzinger et al. 2017), besteht in diesem Strukturtyp großes Potenzial für Maßnahmen an der Oberfläche.

Gebiete mit mittlerer Versiegelung und Dichte: Charakteristische Bauformen in reinen Wohngebieten sind dichtere Einfamilienhäuser, Doppelhäuser, Reihenhäuser als Ein- und Mehrfamilienhäuser mit 1 bis 2 Geschossen. Teilweise finden sich auch betriebliche Gebäude sowie Handels- oder Dienstleistungsgebäude mit den dazugehörigen Stellplätzen.

Bei Gebieten mittlerer Versiegelung und Dichte ist die Flächenverfügbarkeit nicht bebauter Fläche mit 70 bis $50 \%$ theoretisch noch relativ hoch. Die Grünflächen bieten Potenzial für den Einsatz von NW zur Bewässerung. Auch in diesem Strukturtyp überwiegt eine geringe Verschmutzung der Oberflächen. Jedoch ist eine vermehrte Verschmutzung durch Stellplätze besonders bei Mischnutzung mit einem häufigeren Stellplatzwechsel möglich. Da es potenziell eine große Anzahl an EigentümerInnen gibt, besteht für die Umsetzung großflächiger Maßnahmen ein hoher Abstimmungsbedarf.

Die theoretische Flächenverfügbarkeit wird durch zusätzliche versiegelte Flächen weiter eingeschränkt. Unversiegelte Flächen stellen je nach Bebauungstyp nur noch zwischen 10 und $45 \%$ der Gesamtfläche (Buchholz und Kossmann 2015). In diesen Gebieten kann die Flächenverfügbarkeit den Einsatz dezentraler Maßnahmen einschränken. Jedoch ist auch der Anteil an Flachdächern oft höher, welche den Einsatz von Gründächern ermöglichen (Kruse et al. 2014).
Gebiete mit mehrgeschossiger Bebauung mit mittlerer Versiegelung und hoher Dichte: Bauformen in diesen Gebieten umfassen überwiegend mehrgeschossige Wohngebäude, Zeilenbauten, Wohnhausanlagen, offene Blockrandstrukturen sowie geschlossene Bebauung im Bereich von Innenstädten. In Kerngebieten finden sich weiters auch betriebliche Gebäude, Handelsoder Dienstleistungsgebäude sowie öffentliche Einrichtungen mit den dazugehörigen Stellplätzen.

Die mittlere Flächenverfügbarkeit bietet Potenzial für viele NWB-Maßnahmen. Weiters kann das NW sowohl als Brauchwasser oder für die Bewässerung genutzt werden. Bei mehrgeschossigen Wohnbauten mit einem/r Eigentümer/in besteht eine große Wirkmächtigkeit, welche die Umsetzung erleichtern kann. Die Flächen, welche für die innere Erschließung benötigt werden, können eine Restriktion darstellen, da die unversiegelte Fläche geringer ausfällt. Durch den Stellplatzbedarf kann weiters eine höhere Verschmutzung des Oberflächenabflusses vorliegen, welche den Einsatz von Versickerungsmaßnahmen einschränkt.

Teilweise vorhandene Flachdächer stellen eine Möglichkeit für den Einsatz von Gründächern dar. Weiters finden sich in diesem Strukturtyp teilweise große zusammenhängende Grünflächen, welche ein großes Potenzial für die dezentrale NWB bieten. Da diese jedoch gerade bei Neubauten oft über Tiefgaragen liegen, kann die Anwendbarkeit von Versickerungsanlagen eingeschränkt sein (Kruse et al. 2014).

Mehrgeschossige Wohngebiete mit hoher Versiegelung und Dichte: Die Bauformen umfassen offene sowie geschlossene Blockrandstrukturen, dichte Mehrfamilienhäuser mit einem geringen Freiflächenanteil und dicht bebaute Strukturen in Wohngebieten.

Für diesen Strukturtyp stellen vor allem die Nutzung des NW als Brauchwasser und der geringe Abstimmungsbedarf bei wenigen EigentümerInnen Potenziale für den Einsatz von NWBMaßnahmen dar. Aufgrund der geringen Flächenverfügbarkeit ist der Einsatz von NWB-Maßnahmen mit einem großen Flächenbedarf eingeschränkt. Zusätzlich ist mit einer größeren Anzahl an Stellplätzen zu rechnen, welche einen negativen Einfluss auf die Qualität des Oberflächenabflusses haben können. 
Auch in diesem Strukturtyp können vorhandene Flachdächer ein Potenzial darstellen. Die ohnehin geringe unversiegelte Fläche kann jedoch ebenfalls durch Tiefgaragen den Einsatz von Versickerungsanlagen weiter einschränken (Kruse et al. 2014).

Bebauung mit hoher Versiegelung, Dichte und Mischnutzung: Charakteristisch für diesen Strukturtyp ist eine sehr dichte, geschlossene Bebauung entlang der Straßen im Bereich von Stadtzentren, Innenstädten und Stadterweiterungsgebieten. Nutzungen umfassen neben dem Wohnen auch Handel, Gewerbe, Gastronomie sowie öffentliche Einrichtungen.

Auch in Stadtzentren besteht aufgrund der hohen Dichte Potenzial für die Nutzung von NW als Brauchwasser. Maßnahmen an der Oberfläche sind durch die geringe Flächenverfügbarkeit, den hohen Nutzungsdruck und die mögliche höhere Verschmutzung durch Stellplätze nur eingeschränkt möglich.

Weitere Restriktionen können sich durch bestehende Tiefgaragen oder den Denkmalschutz (z. B. bei Dachneigung, -material) ergeben. Die Innenhöfe bzw. Flachdächer können die verbleibenden Flächenpotenziale in diesen Gebieten darstellen (Kruse et al. 2014).

Öffentliche Einrichtungen und Sondergebiete: Dieser Typ umfasst Sondernutzungen und öffentliche Einrichtungen, deren Standorte über die Flächenwidmung einem bestimmten Zweck zugewiesen sind, wie etwa Schulen, Kindergärten, kulturelle Einrichtungen, Behörden und Ämter, soziale Einrichtungen, Veranstaltungszentren, Kasernen oder Sportanlagen.

Bei öffentlichen Einrichtungen spielt vor allem der große individuelle Handlungsspielraum eine große Rolle. Wenn der Einsatz dezentraler NWB-Maßnahmen in einer Gemeinde angestrebt wird, kann durch die Umsetzung auf öffentlichen Grundstücken eine Vorbildwirkung erzielt werden, welche zur Bewusstseinsbildung beiträgt (Matzinger et al. 2017). Da sich die Nutzungen stark unterscheiden, kann es jedoch besonders durch große Stellplätze zu einer Einschränkung beim Einsatz von Versickerungsmaßnahmen durch die höhere Verschmutzung kommen. Je nach Einrichtung können Potenziale der Regenwassernutzung zur Bewässerung von Grünflächen bzw. zur Nutzung als Brauchwasser bestehen.
Büro, Verwaltung, Handel und Gewerbe: In diesem Strukturtyp gibt es keine Wohnnutzung. Die Bauformen variieren je nach konkreter Nutzung, charakteristisch ist jedoch der zumeist hohe Bedarf an Stellplätzen. Daten zu diesen Nutzungen stammen vor allem aus dem GWR und umfassen sowohl die Einwohnerdichte als auch unterschiedliche Gebäudeeigenschaften.

Bei diesem Strukturtyp besteht ebenfalls Potenzial für die NW-Nutzung als Brauchwasser, jedoch ist es abhängig vom Bedarf, der sich nach der Anzahl an Beschäftigten richtet. Bei der Flächenverfügbarkeit kann von einer zusätzlichen Versiegelung durch Stellplätze ausgegangen werden, welche eine Restriktion darstellen. Ab 75 Stellplätzen bei geringem Fahrzeugwechsel bzw. 20 Stellplätzen bei häufigem Fahrzeugwechsel kommt es zu einer Einschränkung beim Einsatz von Versickerungsmaßnahmen (ÖWAV-Regelblatt 45 2014). Da in diesen Gebieten in der Regel viele der Gebäude mit Flachdächern ausgeführt sind, stellen diese relevante Flächenpotenziale dar (Kruse et al. 2014).

Betriebsgebiete und Industrie: Darunter fallen Flächen, die als Betriebsgebiet und Industriegebiet gewidmet und genutzt sind. Auch Betriebsflächen aus der DKM können diesem Typ zugeordnet werden, diese beinhalten beispielsweise die befestigten bzw. versiegelten Nebenflächen von Gewerbebetrieben oder Bauernhöfen.

Aus den abgegrenzten Kriterien lassen sich für Betriebsgebiete und Industrie keine Potenziale direkt ableiten. Es sind bei produzierenden Betrieben hohe Verschmutzungsgrade bzw. Kontaminationen von Böden möglich, welche eine oberflächliche Ableitung und Versickerung stark einschränken. Zusätzlich wird bei diesem Strukturtyp von einer hohen Versiegelung durch Betriebsflächen ausgegangen, welche die Flächenverfügbarkeit mindert.

$\mathrm{Da}$ in diesen Gebieten in der Regel viele der Gebäude mit Flachdächern ausgeführt sind, stellen diese relevante Flächenpotenziale dar (Kruse et al. 2014). Betriebs- oder Industriegebiete sind oft mit Grüngürteln von anderen Nutzungen abgetrennt, auch diese können relevante Flächen für die NWB beinhalten. Die Anwendbarkeit von NWB-Maßnahmen hängt bei diesen Gebieten vor allem von der Art bzw. den Emissionen der jeweiligen Betriebe ab (ÖWAV-Regelblatt 35 2003; ÖWAVRegelblatt 45 2014).

Dörfliche Gebiete mit landwirtschaftlichen Betrieben: Darunter fallen jene dörflichen Bereiche, in welchen sich landwirtschaftliche Hofstellen befinden. Diese Flächen sind als Agrargebiete bzw. Dorfgebiete gewidmet, aktuelle Informationen über bestehende landwirtschaftliche Betriebe können über INVEKOS-Daten ergänzt werden.

Bei diesem Strukturtyp wird von einem Potenzial zur NW-Nutzung für Bewässerung und als Brauchwasser ausgegangen. Zusätzlich stellen wenige EigentümerInnen ein zusätzliches Potenzial aufgrund ihrer hohen individuellen Handlungsspielräume dar. Da mit versiegelten Hofflächen gerechnet wird, ist auch die Flächenverfügbarkeit gemindert. Bei aktiven landwirtschaftlichen Betrieben ist zusätzlich der Einsatz von Versickerungsanlagen für den Abfluss von Hofflächen aufgrund deren Verschmutzung stark eingeschränkt.

\section{Diskussion und Ausblick}

Aus der herangezogenen Literatur konnte eine Methodik für die Abgrenzung siedlungswasserwirtschaftlich relevanter Strukturtypen erarbeitet werden. Mit den vier gewählten Kriterien wurden neun Strukturtypen abgegrenzt. Diese sind mit Strukturtypen vergleichbar, welche in der Literatur zu finden sind (Kruse et al. 2014; Müller et al. 2018). Die beschriebenen Strukturtypen können jedoch im Gegensatz zu bereits publizierten auf Basis von flächendeckend in Österreich verfügbaren amtlichen Daten abgebildet werden. Eine österreichweite Anwendung der beschriebenen Methode zur Abgrenzung ist somit möglich.

Restriktionen in der Anwendung der hier beschriebenen Methode bestehen jedoch vor allem aufgrund der heterogenen Datenbasis und Datenverfügbarkeit in den österreichischen Bundesländern. Zum einen hängen die Ergebnisse der Abgrenzung von der Aktualität bzw. Qualität der Eingangsdaten ab. Zum anderen ist eine Abgrenzung mancher Merkmale, wie beispielsweise die gesamte versiegelte Fläche, mittels der vorhandenen Daten nicht möglich.

Um diesem Problem entgegenzuwirken, könnte die Datengrundlage mithilfe automatisierter Auswertungen von Luftbildern verbessert werden. Diese kann genutzt werden, um versiegelte Nebenflächen besser abschätzen zu 
können. Eine mögliche Datengrundlage stellt hierbei die European Settlement Map dar, die in einer Auflösung von 2,5 m vorliegt (EC 2017). Zukünftige Erweiterungen können beispielsweise in Anlehnung an methodische Zugänge im Bereich der Stadtklimatologie (Müller et al. 2018) erfolgen.

Die Potenziale und Restriktionen der einzelnen Strukturtypen konnten mithilfe der Kriterien und Indikatoren zunächst grob abgeschätzt und daraufhin anhand publizierter Studien spezifiziert werden. Es zeigen sich zum Teil deutliche Unterschiede zwischen den Strukturtypen in der potenziellen Anwendbarkeit unterschiedlicher NWB-Maßnahmen. Die Beurteilung der Anwendbarkeit bezieht sich beim derzeitigen Stand primär auf die unterschiedlichen Prozesse der NWB. Eine Zuordnung des Umsetzungspotenzials einzelner NWB-Maßnahmen $\mathrm{zu}$ den Strukturtypen ist in einem weiteren Schritt notwendig, um die Entscheidungsfindung besser zu unterstützen.

Aus den zuvor beschriebenen Potenzialen und Restriktionen lassen sich bei einer Adaptierung der NWB prioritär zu betrachtende Strukturtypen ableiten.

- Besonders großes Potenzial für die Umsetzung von dezentralen NWBMaßnahmen haben öffentliche Einrichtungen und Sondergebiete sowie Gebiete mit mehrgeschossiger Bebauung mit mittlerer Versiegelung und hoher Dichte. Diese Strukturtypen sollten in der Planung dezentraler NWB-Maßnahmen zu Beginn betrachtet werden.

- Weiteres Potenzial bieten die Strukturtypen Büro, Verwaltung, Handel und Gewerbe, Mehrgeschossige Wohngebiete mit hoher Versiegelung und Dichte sowie Betriebsgebiete und Industrie, wenn die Flächenverschmutzung gering ist. Diese stellen aufgrund der hohen Wirkmächtigkeit der EigentümerInnen ebenfalls wichtige Strukturtypen bei der Umsetzung dezentraler NWB-Maßnahmen dar.

- Gebiete mit geringer und mittlerer Versiegelung und Dichte haben zwar ein großes Potenzial aufgrund der geringen Verschmutzung und der Flächenverfügbarkeit, die Wirkmächtigkeit des Einzelnen ist jedoch eingeschränkt. Diese Gebiete sind aber politisch wertvoll, da hier viele Menschen erreicht werden können.
Aufgrund der Generalisierung auf Baublockebene ergeben sich jedoch bei der Bewertung Potenziale und Restriktionen der Strukturtypen Herausforderungen. Die Probleme bei der Abbildung der tatsächlich versiegelten Flächen wurden bereits diskutiert. Zusätzlich stellt besonders die generelle Bestimmung der Qualität des Oberflächenabflusses eine Einschränkung dar.

$\mathrm{Zu}$ den wichtigsten Merkmalen auf Baublockebene, bei der Beurteilung der Qualität des Oberflächenabflusses, gehören die Größe und das Material von Dächern, die Anzahl an Stellplätzen und die Häufigkeit des Fahrzeugwechsels sowie der Verschmutzungsgrad von Hofflächen. Je nach Merkmal erfolgt, wie bereits beschrieben, eine Zuordnung zu einer Flächenkategorie, welche die Anwendbarkeit dezentraler NWBMaßnahmen beeinflusst. Besonders bei Flächen ab der Kategorie F3 ergeben sich größere Einschränkungen bei der Wahl von Versickerungsmaßnahmen, durch eine notwendige Reinigung des NW (ÖWAV-Regelblatt 35 2003; ÖWAVRegelblatt 45 2014). Diese Merkmale lassen sich jedoch nur indirekt oder gar nicht durch die gewählten Kriterien abbilden und einzelne Flächen höherer Kategorien lassen sich aufgrund der Aggregation nur schwer abgrenzen. Diesem Problem könnte mit einer Ergänzung der Daten durch Vor-OrtErhebungen entgegengewirkt werden. Die dadurch gewonnen Informationen können auch für die siedlungswasserwirtschaftliche Planung und Modellierung des Systems herangezogen werden (Muschalla et al. 2015). Eine detaillierte Erhebung der Daten kann jedoch in einem weiteren Schritt auch eine weitere Unterteilung der Strukturtypen erfordern.

Kruse et al. (2014) haben in ihrer Abgrenzung auch eine Erweiterung der Merkmale um sozio-ökonomische Kriterien vorgeschlagen. Diese könnten zum Beispiel die finanzielle Machbarkeit durch die EigentümerInnen widerspiegeln. Eine flächendeckende Bewertung dieser mittels der herangezogenen Daten ist jedoch nicht möglich.

Zusätzlich wird in den ÖWAV-Regelblättern auch auf die Verschmutzung von Verkehrsflächen eingegangen (ÖWAV-Regelblatt 35 2003; ÖWAV-Regelblatt 45 2014). Obwohl Verkehrsflächen eine wichtige Rolle bei der NWB spielen, wurden diese aus der präsentierten Analyse ausgespart, da die notwendige Datengrundlage und die
Bewertung dieser signifikant von der Baublockeben abweicht. Eine Erweiterung der Methodik um eine räumliche Analyse stellt jedoch einen möglichen weiteren Schritt dar.

Bei der Erstellung der Methodik standen bestehende Siedlungsstrukturen im Vordergrund. Die Erkenntnisse können jedoch auch auf Neubaugebiete übertragen werden. Für die Planung von Stadt- und Siedlungserweiterungen gilt es, die Möglichkeiten im Rahmen von Flächenwidmung und Bebauungsplan auszuschöpfen, die benötigten Flächen abzusichern und eine Behandlung des NW entsprechend dem aktuellen Stand von Technik und Forschung vorzusehen. Je nachdem, in welchen Typen die Erweiterungen stattfinden, können die präsentierten Potenziale und Restriktionen einen Rahmen zur gebietsspezifischen Auswahl von dezentralen NWB-Maßnahmen bieten.

Um die Praxistauglichkeit der Methode $\mathrm{zu}$ testen, wird diese in einem weiteren Schritt im Rahmen einer Fallstudie im Abwasserverband an der Traisen am Beispiel der Stadt St. Pölten angewandt und weiterentwickelt. Die Strukturtypen sowie ihre Potenziale und Restriktionen sollen als Input für die Entscheidungsfindung und Standortwahl dienen und in Workshops mit Verwaltung und Abwasserverband diskutiert und erweitert werden.

\section{Schlussfolgerungen}

Eine Methodik zur Abgrenzung von Strukturtypen für die NWB auf Basis bestehender amtlicher Daten in Österreich wurde in diesem Artikel aufgezeigt. Anhand von vier Merkmalskategorien konnten neun Strukturtypen unterschieden werden. Weiters wurden die Potenziale und Restriktionen in Bezug auf den Einsatz von dezentralen NWB-Maßnahmen analysiert.

Auf Basis von Strukturtypen können im Bestand jene Bereiche identifiziert werden, in welchen es besondere Potenziale bzw. Restriktionen aus Sicht der NBW gibt. Darüber hinaus bieten die Instrumente der örtlichen Raumplanung Möglichkeiten, den Umgang mit NW im Rahmen von Entwicklungskonzepten, Flächenwidmung und Bebauungsplan vorausschauend in die Gemeindeentwicklung zu integrieren. Insbesondere auf Ebene des Bebauungsplans gibt es zahlreiche Möglichkeiten, die Versickerung bzw. Ableitung von NW, die Versiegelung von Flächen 
oder den Ausbau und die Ausgestaltung von öffentlichen und privaten Verkehrsflächen zu bestimmen. Es wird somit gezeigt, dass sowohl für bestehende Siedlungsgebiete als auch bei zukünftigen Erweiterungsflächen eine integrative Herangehensweise von Siedlungswasserwirtschaft und Raumplanung Potenziale für die Planung dezentraler NWB-Maßnahmen bietet.

Danksagung Die Entwicklung dieser Methode ist im Zuge der Projekte „FlexAdapt - Entwicklung flexibler Ad- aptierungskonzepte für die Siedlungsentwässerung der Zukunft“, gefördert durch das Ministerium für Nachhaltigkeit und Tourismus (Projektnummer B600624), und „Siedlungswasserwirtschaftliche Analyse räumlicher Strukturen - Fallstudie Abwasserverband an der Traisen (AWV_Traisen)“, gefördert durch den Abwasserverband an der Traisen, entstanden.

Funding Open access funding provided by University of Natural Resources and Life Sciences Vienna (BOKU).
Open Access Dieser Artikel wird unter der Creative Commons Namensnennung 4.0 International Lizenz (http:// creativecommons.org/licenses/by/4. $0 /$ deed.de) veröffentlicht, welche die Nutzung, Vervielfältigung, Bearbeitung, Verbreitung und Wiedergabe in jeglichem Medium und Format erlaubt, sofern Sie den/die ursprünglichen Autor(en) und die Quelle ordnungsgemäß nennen, einen Link zur Creative Commons Lizenz beifügen und angeben, ob Änderungen vorgenommen wurden.

Literatur

ARL (2017): ARL Forschungskonzept 2017-2022. Hannover: Verlag der Akademie für Raumforschung und Landesplanung. https://shop.arlnet.de/media/direct/pdf/forschung/forschungs konzept_2017-2022.pdf

Assmann, M., Dürr, K., Haberfellner-Veit, E., Laber, J., Lindtner, S., \& Tschiesche, U. (2015): Branchenbild der österreichischen Abwasserwirtschaft 2016. Wien: Österreichischer Wasserund Abfallwirtschaftsverband (ÖWAV) \& Kommunalkredit Public Consulting (KPC). https:// www.umweltfoerderung.at/fileadmin/user upload/media/umweltfoerderung/Dokumente Betriebe/Wasser_Betriebe/Studien_Wasserwirt schaft/Branchenbild_2016.pdf

Braubach, M., Egorov, A., Mudu, P., Wolf, T. Ward Thompson, C., \& Martuzzi, M. (2017): Effects of Urban Green Space on Environmental Health, Equity and Resilience. In N. Kabisch, H. Korn, J. Stadler, \& A. Bonn (Hrsg.), Nature-Based Solutions to Climate Change Adaptation in Urban Areas: Linkages between Science, Policy and Practice (S. 187-205). Cham: Springer International Publishing. https://doi.org/10.1007/9783-319-56091-5_11

Buchholz, S., \& Kossmann, M. (2015): Research note. Visualisation of summer heat intensity for different settlement types and varying surface fraction partitioning. Landscape and Urban Planning, 144, 59-64. https://doi.org/10.1016/j. landurbplan.2015.08.002

Buchholz, S, Kossmann, M., \& Roos, M. (2016) INKAS - a guidance tool to assess the impact of adaptation measures against urban heat. Meteorologische Zeitschrift, 25(3), 281-289. https:// doi.org/10.1127/metz/2016/073

Burns, M. J., Fletcher, T. D., Walsh, C. J., Ladson, A. R., \& Hatt, B. E. (2012): Hydrologic shortcomings of conventional urban stormwater management and opportunities for reform Landscape and Urban Planning, 105(3), 230-240. https://doi.org/10.1016/j.landurbplan.2011.12. 012

DWA \& BWK (Hrsg.) (2016): Grundsätze zur Bewirtschaftung und Behandlung von Regenwetterabflüssen zur Einleitung in Oberflächengewässer (Entwurf Oktober 2016). Hennef: Deutsche Vereinigung für Wasserwirtschaft, Abwasse und Abfall.

EC (2017): European Settlement Map 2017. Abgerufen von http://land.copernicus.eu/paneuropean/GHSL/european-settlement-map/ esm-2012-release-2017-urban-green/view

EN 752 (2017): Entwässerungssysteme außerhalb von Gebäuden.

Fletcher, T. D., Shuster, W., Hunt, W. F., Ashley,

R., Butler, D., Arthur, S., Trowsdale, S., Barraud,
S., Semadeni-Davies, A., Bertrand-Krajewski, J.-L., Mikkelsen, P. S., Rivard, G., Uhl, M., Dagenais, D., \& Viklander, M. (2015): SUDS, LID, BMPs, WSUD and more - The evolution and application of terminology surrounding urban drainage. Urban Water Journal, 12(7), 525-542. https://doi.org/10.1080/1573062X.2014.916314 Gasienica-Wawrytko, B., Hagen, K., Trimmel, H., \& Tötzer, T. (2014): Kapitel 6: Charakterisierung der Stadtraumtypen. In R. Stiles, B. Gasienica-Wawrytko, K. Hagen, H. Trimmel, W. Loibl, M. Köstl, T Tötzer S. Pauleit, A Schirmann, \& W. Feilmayr, Urban Fabric Types and Microclimate Response - Assessment and Design Improvement. Final Report. Wien. http://urbanfabric.tuwien.ac.at/index.php/de/ ergebnisse-endbericht

Grimm, K., \& Achleitner, M. (2010): Naturnahe Oberflächenentwässerung für Siedlungsgebiete - Leitfaden für die Planung. St. Pölten Amt der NÖ Landesregierung Gruppe Wasser. www noe gvat/noe/Wasser/Naturnahe Oberflaechenentwaesserung_-_Leitfaden_fuer_ die_Pla.pdf

Hegger, M., Dettmar, J., Martin, A., Meinberg, T. Boczek, B., Drebes, C., Greiner, M., Hesse, U. Kern, T., Mahlke, D., Al Najjar, A., Schoch, C. Schulze, J., Sieber, S., Stute, V., Sylla, O., Wurzbacher, S., \& Zelmer, A. (2012): UrbanReNet I Vernetzte regenerative Energiekonzepte im Siedlungs- und Landschaftsraum.

Heiland, S., Mengel, A., \& Hänel, K. (2017): Bundeskonzept Grüne Infrastruktur: Fachgutachten. Bonn-Bad Godesberg: Bundesamt fü Naturschutz

Kruse, E., Zimmermann, T., Kittel, A., Dick haut, W., Knieling, J., \& Sörensen, C. (2014): Stadtentwicklung und Klimaanpassung - Klimafolgen, Anpassungskonzepte und Bewusstseinsbildung beispielhaft dargestellt am Einzugsgebiet der WANDSE. Hamburg: Staats- und Universitätsbibliothek Hamburg Carl von Ossietzky. edoc.sub.uni-hamburg.de/klimawandel/files/ 986/Band+2_Stadtentwicklung+Klimaanpas

sung Wandse.pdf

Kuzmich, F., Kemstock, S., Kleindienst, G., Neudecker, L., Zlonicky, P., Eder, B., Luchsinger, C., Finka, M., Bucek, S., Husár, M., Bláha, P., Jamecný, L., Chvostalová, I., Müller, C., Bucek, M., \& Ondrejicka, V. (2011): Siedlungsformen für die Stadterweiterung. Magistratsabteilung 18 Stadtentwicklung und Stadtplanung, Magistratsabteilung der Koordinierung der Gebietssysteme Magistrat der Hauptstadt der SR Bratislava.

Matzinger, A., Riechel, M., Remy, C., Schwarz müller, H., Rouault, P., Schmidt, M., Offermann, M., Strehl, C., Nickel, D., Sieker, H.,
Pallasch, M., Köhler, D., Möller, C., Büter, B., Leßmann, D., von Tils, R., Säumel, I., Pille, L, Winkler, A, Bartel, $H$, Heise, $S$, Heinzmann, B., Jowig, K., Rehfeld-Klein, M. \& Reichmann, B. (2017): Zielorientierte Planung von Maßnahmen der Regenwasserbewirtschaftung - Ergebnisse des Projektes KURAS Berlin. http://kuras-projekt.de/downloads/ erzeugnisse-regenwasserbewirtschaftung/ Müller, N., Hecht, R., \& Buchholz, S. (2018): Bebauungsstrukturklassifikation NRW - Grundlage für Klimamodellsimulationen. In: Meinel, Gotthard; Schumacher, Ulrich; Schwarz, Steffen; Richter, Benjamin (Hrsg.): Flächennutzungsmonitoring IX: Nachhaltigkeit der Siedlungs- und Verkehrsentwicklung?. Berlin: Rhombos-Verlag, 2017, (IÖR-Schriften; 73), S. 81-91

Muschalla, D., Sulzbacher, R. M., Leimgruber, J., Maier, R., Ertl, T., Neunteufel, R., Kretschmer, F., Kleidorfer, M., \& Tscheikner-Gratl, F. (2015): Auf effizientem Wege von den Daten zum Model (DATMOD) - Sanierungs- und Anpassungsplanung von kleinen und mittleren Kanalnetzen Wien, Österreich: Bundesministerim für Landund Forstwirtschaft, Umwelt und Wasserwirtschaft.

NÖ ROG (2014): Niederösterreichisches Raumordnungsgesetz. LGBl. Nr. 3/2015 i.d.g.F. LGBl. Nr. 65/2017

ÖROK (2013): ÖROK Atlas. Wien: Österreichische Raumordnungskonferenz. https://www. oerok-atlas.at/\#indicator/72

ÖWAV-Regelblatt 35 (2003): Behandlung von Niederschlagswässern. Wien: Österreichischer Wasser- und Abfallwirtschaftsverband.

ÖWAV-Regelblatt 45 (2014): Oberflächenentwässerung durch Versickerung in den Untergrund. Wien: Österreichischer Wasser- und Abfallwirtschaftsverband.

Stewart, I. D., \& Oke, T. R. (2012): Local Climate Zones for Urban Temperature Studies. Bulletin of the American Meteorological Society, 93(12), 1879-1900. https://doi.org/10.1175/BAMS-D11-00019.1

Stoeglehner, G., Neugebauer, G., Erker, S., \& Narodoslawsky, M. (2016): Integrated Spatial and Energy Planning. Cham: Springer International Publishing. https://doi.org/10.1007/9783-319-31870-7

Woods Ballard, B., Wilson, S., Udale-Clarke, $H$. Illman, S., Scott, T., Ashley, R., \& Kellagher, R. (2015): The SuDS Manual. London, UK: CIRIA, C753. Abgerufen von http://www.susdrain.org/ resources/SuDS_Manual htm 